\title{
SECURE COMMUNICATION MECHANISMS FOR GSM NETWORKS
}

\author{
Chi-Chun Lo and Yu-Jen Chen \\ Institute of Information Management \\ National Chiao-Tung University \\ 1001 Ta Hsueh Road \\ Hsinchu, Taiwan 300, ROC \\ Email: cclo@cc.nctu.edu.tw
}

\begin{abstract}
With the advance of wireless communications technology, mobile communications has become more convenient than ever. However, because of the openness of wireless communications, how to protect the privacy between communicating parties is becoming a very important issue. In this paper, we fucus on the security of the Global System for Mobile communication (CSM) networks. A secure communication architecture for the GSM network is proposed. In the proposed architecture, we use public-key cryptography for user authentication and stream cipher for message encryption and dectyption. An authentication protecel and a key generation method are presented in conjunction with the propesed architecture. Cryptanalysis and operational analysis show that the authentication protocol is secure and efficient. Simulation results indicate that the key generation method can always produce key strings of evenly distributed 0's and l's and with infinite period.
\end{abstract}

Keywords: GSM Networks, Wireless Communications, Mobile Communications, Block Cipher, Stream Cipher, Authentication

\section{INTRODUCTION}

Mobile communications has become morc popular and easier for the past few years. Nowadays, people can communicalc with each other on any place at any time. However, llic openness of wircless communications poses scrious sccurity threats to communicating partics. How to provide secure communication channels is essential to the success of a mobilc communication nctwork. The Global System for Mobilc communications (GSM) is the standard for digital mobile communications. It has a comprehensive set of security features [6]. However, these features have several limitations. In this paper, we propose a securc communication architecture for GSM networks. In the proposed architecture, public-kcy cryptography and strcam cipher are used for uscr authentication and message encryption/ decryption, respectively. An authentication protocol and a kcy gencration method are presented in conjunction with the proposed architecture. Cryptanalysis and operational analysis show that the authentication protocol is secure and efficient. Simulation results indicate that the key generation method can always produce key strings of evenly distributed 0 's and l's and with infiutc period. In the following section, litcrature review is presented. Section 3 first describes the proposed architecture, and then details the authentication protocol and the key generation method. Analysis is given in Section 4. Section 5 concludes this paper with possible future rescarch directions.

\section{LITERATURE REVIEW}

\subsection{GSM [6][18]}

The GSM is the first mobile communication system which has comprehensive security featurcs. It is gaining tremendous supports from the telecommunication industry.
The security architecture of GSM is intended to prevent unanthorized network access, disallow subscriber impersonation, protect conlidentiality, and provide privacy. GSM's sccurily services include anonymity, authentication. signaling protection, and user data prolection.

Fig. I depicts the sccurity architecture of CSM. It is composed of threc ticrs. The first tier; namely, the $A 3$ algorithm, uses the challenge-response method $|1|$ for user authentication. The sccond tier, namely, the A8 algorithm, uses the output from the A3 algorithm to generate the key string for the AS algorithm. The last tier; namely, the A5 algorithm, uses a proprictary algorithm for message encryption/decryption. This archirceture has the following problems: challenge-response is a simple authentication method, but is not very secure [4]; the length of the key generated by the $A 8$ algorithm is fixed ( 114 bits), hence this key might be disclosed by the brute-force altack [1]; the $A 3$, $A 8$ and $A 5$ algorithus are proprietary, thus their security can not be easily verified; and the $A 5 / 1$ algorithm is subject to cxport control.

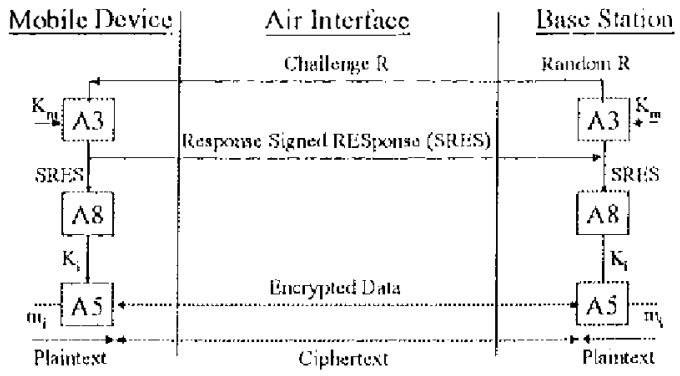

Fig. 1. Security Architecture of GSM

\subsection{Stream Cipher $[20]$}

Symmetric cryptosystems are further classified into

Contributel P'alser 
block cipher and stream cipher. Block cipher divides the plaintext into blocks and encrypts cach block independently. On the other hand, stream cipher encrypts the plaintext on a bit-by-bit (or byte-by-byte) basis. (n essence, GSM networks carry mostly voice, which is one type of continuous data. Since stream cipher is based on the eXclusive OR (XOR) operation, to cncrypt/decrypt voice data bit-by-bit (or bytcby-byte) using stream cipher is vety efficient. In general, streain cipher is much simpler and faster than block cipher [1][20]. Therefore, strcam cipher is a good choice for message encryption/decryption for GSM networks.

The major problem of strcam cipher cryptography is the difficulty of gencrating a long unpredictable bit pattern (keystream). In the onc-time pad of stream cipher, a keystream is a sequence of randomly generated bits, and the probability of one bit to be 1 , independent of other bits, is cqual to one half. An idcal kcystream in one-time pad is purely randoin and has infinite length. The keystream can not be gencrated by the receiving end, and can not be distributed to the receiving end cither. The pscudorandom bit generator [20] has becn widely used to construct the keystream. It generates a fixed-length pseudorandom noise as the keystrcam. How to increase the length of the kcystream while still maintaining its randomness is important to the security of stream cipher.

\subsection{The X.509 Threc-way Exchange [21]}

The X.509 three-way cxchange, described in ITU-T Recommendations X.5(2), is a novel example of a public-key based authentication protocol. Kcy management of this protocol is securer than those of the protocols using symmetric cryptography. However, the X.509 three-way cxchange has some noticcable problems. Since the X.509 three way cxchange performs encryption before signiug, the attacker may remove the signature from the encrypted message and replace it with his own [2]. Futhermore, the $X .509$ three-way exchange does not provide perfect forward secrecy so that the disclosure of the private key may compromise the scssion key.

\subsection{Security Attacks}

Security mechanisms are subject to different attacks. Following is a discussion on some most noticcable attacks.

\subsubsection{Replay Attack [1]}

An attacker can use this attack to capture legitimate messages and retransmit them for illegal purpose. T'o defeat such an attack, non-repeated random numbers arc oflen used to ensure that all replayed messages will be detected. Time stamp, sequence number, and challenge-response are threc different types of nonces. Each has its own limitations. As for time stamp, it is very difficult and expensive to maintain clock-synchronization among all partics at all time; as for sequence number, it requires that each party has to maintain state information of other parties, which is impractical in an open systcin [8]; as for challenge-response, its security depends on the randomress of nonce, and it necds an cxtra message exchange to complete mutual authentication.

\subsubsection{Guessing Attack [1]}

Autlucntication using password is widely used by many security systems. However, password is vul nerable under the dictionary attack by which an attacker can guess the password successfully. Public-key cryptography provides a means for preventing the guessing attack.

\subsubsection{Interleaving Attack [5]}

If an attacker could collect information from different cxecutions of a security protocol, he/she might be able to break the protocol. This is the so-called interlcaving attack. Based on the paper given by Ray Bird et. al [5], interleaving attacks include the known plaintext attack [5], the chosen ciphertext attack [5], the oracle session attack [4] and the parallel session attack [4]. The cipher block chaining (CBC) cncryption and public-key cryptography are often used to prevent this attack [5].

\subsubsection{Man-in-the-Middle Attack [1]}

An attacker can use the man-in-the-middle atlack to intervenc between two conmunicating partics and mascluerade as one to communicate with another bidirectionally. Public-key cryptosystem using certificate often provides a selution for preventing this attack.

\section{SECURE COMMUNICATION MECHANISMS}

The following notation is defined.

\begin{tabular}{|c|c|}
\hline \multicolumn{2}{|l|}{ Notation: } \\
\hline Identily $_{x}$ & X's identity \\
\hline$K_{\text {priv. }}$ & X's private kcy \\
\hline $\mathrm{K}_{\text {putb, }}$ & X's public kcy \\
\hline$S_{x}\{\ldots\}$ & X's signature followed by data \\
\hline$S^{\prime} x\{\ldots\}$ & $X$ 's signature \\
\hline$E_{K p u t 1, x}()$ & message cncrypted by X's public key \\
\hline $\operatorname{Cort}(X)$ & $\begin{array}{l}\text { X's certificate; it contains X's identity, } \\
\text { X's public kcy, and certificate } \\
\text { authority's signature on X's identity and } \\
\text { X's public key }\end{array}$ \\
\hline
\end{tabular}

\subsection{The Proposed Architecture}

The proposed architecture, shown in Fig. 2, still follows the three-ticr structure as described in GSM standards. In this architccture, the C3, C8 and C.5 algorithms are suggested to replace the $A 3, A 8$ and $A 5$ algorithuns, respectively. The lollowing sections detail the $\mathrm{C} 3, \mathrm{C} 8$ and C5 algorithms. 


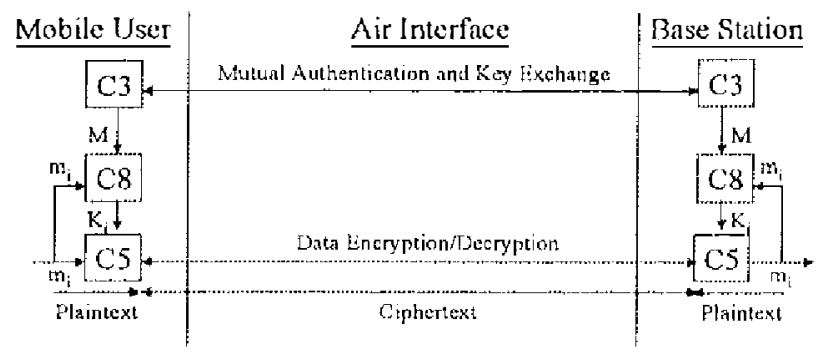

Fig. 2. The Proposce Architecture

\subsection{Authentication Protocol (C3)}

\subsubsection{Design Rationale}

Upon requesting service from a basc station, a mobile user has to prove his/her identity to the base station. After completing the service, he/she is charged for the service he received. Authentication is required not only when a connection is being cstablished but also when the connection is being released. Since a mobile user is charged afterwards, the base station should be able to prevent him from denying the service he received. This implies that non-repudiation is needed. Furthernore, the integrity of the service request message should be preserved. Although public-key cryptography is computationally intensive; however, it docs meet the authentication requirements of GSM networks. According to the discussions aforementioned, the design of C3 refers to the X.509 threc-way exchange. In this design, certificates are required.

\subsubsection{Protocol Design}

The C3 protocol has two phases: the connection phase and the release phase.

\section{Connection Phase}

The connection phase includes three steps. Fig. 3 depicts the connection phase of C3. For mobilc user (MU) m, his/her identity (Identity $\left.{ }_{m}\right)$, private key $\left(K_{\text {priv.ni }}\right)$, public key $\left(K_{\text {pubim }}\right)$ and certificate $(\operatorname{Cert}(\mathrm{m}))$ are issued by certificate authority (CA), and are saved inside the Subscriber Interface Module (SIM) of his/her mobile device. For base station (BS) $s$, its identity (Identity ${ }_{s}$ ), private key $\left(\mathrm{K}_{\text {priv.s }}\right)$, public key $\left(\mathrm{K}_{\text {pub,s }}\right)$ and certificate (Cert(s)) arc also issued by $\mathrm{CA}$. Authentication is performed according to the following procedure:

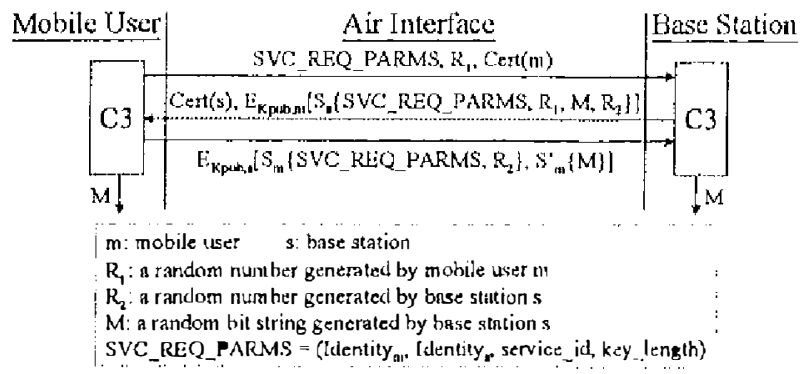

Fig. 3. Connection Phase of the C3 Protocol
Step 1: The MU requests service from the BS. Service request paramelers (SVC_REQ_PARMS) include Identity ${ }_{\mathrm{in}}$, Identity $\mathrm{y}_{\mathrm{s}}$, service_id (the requested service identifier), and key length (the length of the initial kcystream). SVC_REQ_PARMS, along with nonce $R_{1}$ and MU's certificate (Cert(m)), are sent to the BS, where $\mathrm{R}_{\mathrm{l}}$ is a challenge to the BS.

Step 2: The BS uscs Cort(m) to verify MU's identity; generates a random bit string $M$ of length "key_length" and a random number $\mathrm{R}_{2}$, where $\mathrm{R}_{2}$ is a challenge to the MU; signs SVC REQ PARMS, $R_{l}$, $M$, and $R_{2}$, where $R_{1}$ is the response to the MU (The $B S$ is authenticated); uses $K_{\text {put, nt }}$ to encrypt the signed message; sends the encrypted message, along with its certilicate (Cert(s)), to the MU.

Step 3: The MU uses Cert(s) to verify BS's identity; uses $\mathrm{K}_{\text {priv,in }}$ to decrypt the encrypted message rcceived; uses $K_{p u t, s}$ to verify the signature of the BS; cliceks whether SVC REQ PARMS received is the same as the onc he/she sent in Step l; signs $S V C$ REQ PARMS and $R_{2}$, where $R_{2}$ is the response to the BS (The MU is authenticated); signs $M$; uses $\mathrm{K}_{\text {rub, }}$ to encrypt the two signed messages; sends the encrypted message to the BS.

Note that the bit string $M$ is a shared secret between the base station and the mobile user, and is used as the initial keystream of the $\mathrm{C} 8$ algorithm. The base station and the mobile user should negetiate the length of $M$ (key_length) in advance. M's length can be vory long; for cxample, $1 \sim 20$ KBytes which is much longer than the normal kcy length; c.g., 56 bits, 128 bits, and 512 bits.

\section{Release Phase}

The relcase phase, shown in Fig. 4, includes twe steps.

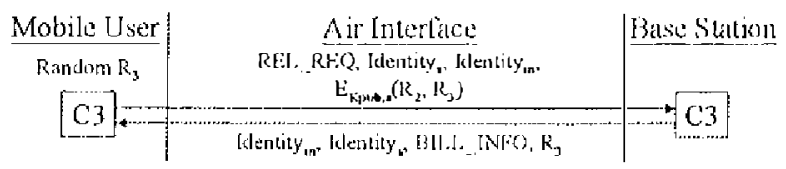

Fig. 4. Relcase Phase of the C3 Protocol

Step 1: The $M U$ generates a random number $R_{3}$, where $R_{3}$ is a clallicnge to the $B S$; uses $K_{p}$ mu, to cricrypt $R_{2}$ and $R_{3}$, where $R_{2}$ is the response to the $B S$ (The $M U$ is authenticated); sends the enerypted message, along with relcase request (REL_REQ), Identity, and Identity ${ }_{\text {sn }}$ to the BS.

Step 2: The BS uses $K_{\text {priv,s }}$ to decrypt the encrypted message reccived; sends $R_{3}$, along with Identity ${ }_{m}$, Identity ${ }_{s}$ and billing information (BILL INFO), to the MU, where $R_{3}$ is the response to the MU (The BS is authenticated).

\subsubsection{Comments}

The securily features of $\mathrm{C} 3$ are highlighted as follows:

- In the first and sccond steps of the conncction phase, certificales. Cert(m) and Cert(s), are used to verify mobile user's and base station's identities, respectively. 
- In the second and third steps of the connection phase, nonces, R1 and R2, are used to provide direct mutual authentication.

- In the first and second steps of the release plase, mutual authentication is provided via $R_{2}$ and $R_{3}$. Notc that $R_{2}$ is reused in the relcase phase with which the mumber of message exchanges in the releasc phase is reduced by one.

- In the second step of the connection phase, the initial keystream (M) of the C8 algorithm, instead of a session key, is exclianged.

- In the second and third steps of the connection phase, signing is performed before encryption.

- In the second and third steps of the connection phase, the service request message is encrypted along with nonce. It is worthy of noticing that the size of the service request message is usually very small. Since the contents of the service request message are limited and casy to predict, an attacker can find probabilistic encryption by guessing enough strings. T' Coil such an attack, it is practical to encrypt the service request message along with an unpredictable random number (nonce) [5].

- In the third step of the connection phase, the service request message is signed by the mobite user; thus, non-repudiation is guarantecd.

- The C3 protocol can provide perject fonward secrecy since the initial kcystream $M$ is not repeated for every scrvice request.

\subsection{Key Generation (CS)}

The C8 algorithm processes input data on a byte-bybyte basis. For an initial kcystrcam of Icngth $k$ bytes, $\left(M_{0}\right.$, $\left.M_{1}, \ldots, M_{j}, \ldots, M_{k-1}\right)$, and an iuput message of Icngth n bytes, $\left(\mathrm{m}_{1}, \mathrm{~m}_{1}, \ldots, \mathrm{m}_{\mathrm{i}}, \ldots, \mathrm{m}_{\mathrm{n}-1}\right)$, the keystream of length $\mathrm{n}$ bytes, $\left(K_{0}, K_{1}, \ldots, K_{i}, \ldots, K_{-1-1}\right)$, is generated according to the following procedure:

Stcp 1: Let $i=0, j=0, N=170, c c=0$;

Step 2: $M_{j}=M_{j}+c c$;

If $j=0$ then $M_{j}=M_{j}$ XOR $N$; else $M_{j}=M_{j}$ XOR $M_{j-1}$; Step 3: $K_{i}=M_{j} ; N=\left(N+m_{l}\right) \bmod 256$

$\mathrm{i}=\mathrm{i}+\mathrm{i} ; \mathrm{j}=\mathrm{j}+\mathrm{l} ; \mathrm{cc}=\mathrm{cc}+\mathrm{l}$;

If $\mathrm{j}=\mathrm{k}$ then resct $\mathrm{j}$ to 0 ;

If $c c=256$ then reset $c c$ to 0 ;

If $\mathrm{i}=\mathrm{n}$, then exit; else goto Step 2 .

where $\mathrm{N}$, an eight-bit string, is used by the XOR opcration to change $M_{0}$ at the beginning of each cycle of $M$; cc is a number added to $M_{j}(j=0$ to $k-1)$ so as to increasc the randomuess of $M_{j}$.

Note that the initial value of $\mathrm{N}$ can be any number between 0 and $255\left(2^{8}-1\right)$. In C8, we use the number $170\left(10101010_{2}\right)$ as the default value of $\mathrm{N}$.

\subsection{Message Encryption/Decryption (C5)}

The C5 algorithm uses strcam cipher for encryption/decryption. In our design, the simplest stream cipher using only the XOR operation [20] is chosen.

\section{ANALYSIS}

\subsection{Analysis of $\mathrm{C3}$}

\subsubsection{Cryptanalysis}

- Kcystrcam (M) revelation

In the second step of the cennection plaase, since the initial keystrcan $M$ is signed by the $B S$, it is impossible for an attacker to obtain $M$. In the third step of the connection phase, $M$ is signed by the MU; thus, it is frec from revelation.

- Interleaving attack

(1) mobile user attack

In the sccond step of the connection phase, if an attacker pretended as the base station, he/she could not sign $\mathrm{R}_{1}$ without base statien's private key. In the second step of the release phase, since an attacker can not decrypt nonce $\mathbf{R}_{3}$ without basc station's private key, he/sle is not able to mascuerade as the base station.

(2) basc station attack

In the first step of the connection phase, if an attacker pretended as the mobile user, he/she could not modify CA's signature on mobile user's identity In the third step of the connection phase, since an attacker can not sign $R_{2}$ without mobile uscr's private key, he/she is not able to pretend as the mobile user. In the first step of the release phase, an altacker call not obtain nonce $\mathrm{R}_{2}$; therefore, it is intpossible for hitu/her to pretend as the mobile user.

- Replay attack

The replay attack is not possible since the challengeresponse mechanism is used.

- Gucssing atuack

The guessing attack is not possible since service request parameters (SVC_RE:_PARMS) are encrypted alung with nonce.

- Man-in-the-middle attack

Both the mobile user and the base station can verify each other's identity by using each other's certificatc. Also, since signing is performed before encryption in the C3 protocol, an altacker can not remove the signature from the encrypted nessage and replace it with its own. Therefere, the man-in-the-middle attack is prevented.

\subsubsection{Operational Analysis}

\section{Number of messige cxchanges}

Nonce $R_{2}$ is used both in the connection phase and the release phase. Hy doing this, the number of messige cxchanges in the release phase is reduced to 2 , which is one less than the minimum number of inessage exchanges required lor a challenge-response based authentication protocol [9]. Consequently, the total number of message exchanges of the $\mathrm{C} 3$ protocol is 5 instead of 6 .

Cryptographic operation

Cryptographic opcration includes 
cncryption/decryption and signing/verification. For comparison, the X.509 three-way exchange is also considered. Table I presents the comparison.

Table 1. Comparison between the X.504 three-way exchange and the $\mathrm{C} 3$ protocol

\begin{tabular}{|c|l|c|c|c|c|}
\hline & \multicolumn{2}{|c|}{ Public-key eryptosystem } & \multicolumn{2}{|c|}{ Signature } \\
\cline { 3 - 6 } & Encryntion & Decryption & Signing & Verification \\
\hline $\begin{array}{c}\text { X.509 } \\
\text { threc-way } \\
\text { exchange }\end{array}$ & Mobile user & 1 & 2 & 1 & 2 \\
\cline { 2 - 6 } & Base station & 2 & 1 & 2 & 1 \\
\hline \multirow{2}{*}{ c3 } & Mobile user & 2 & 1 & 1 & 1 \\
\cline { 2 - 6 } & Basc station & 1 & 2 & 1 & 1 \\
\hline
\end{tabular}

For mobile users, the total number of encryption/decryption of the X.509 three-way cxchange is the same as that of the C3 protocol. However, the C3 protocol performs one more encryption than the X.509 threeway cxchange. For base stations, the total number of cncryption/decryption of the X.509 three-way cxchange is the same as that of the C3 protocol. The X.509 three-way exchange performs one more encryption operation than the C3 protocol. Consequently, base stations using the $\mathrm{C} 3$ protocol are more efficient than those using the $X .50)$ (hrecway cxchangc.

For mobile users, the number of verification of the C.3 protocol is onc less than that of the X.509 threc-way exchange. For base stations, the number of signing of the $\mathrm{C} 3$ protocol is one less than that of the $X .509$ threc-way exchange. With regard to signing/verification, the C3 protocol is more efficient than the $\mathrm{X} .509$ three-way cxchange.

\subsection{Analysis of C8}

\subsubsection{Security Analysis}

The security of the C8 algorithm is evaluated by simulation. The simulator is coded in the $\mathrm{C}$ language and is nunuing on an Intel Pentium II-266MHz. PC with $64 \mathrm{MB}$ RAM. Two metrics, randonmess and period, are sclected for security evaluation. Randonmess is defined to be the probability of being 0 or 1 . For a purcly random keystrcam, the probability of one bit to be 1 , independent of other bits, is equal to one half. Period is defined to be the number of bytes in the repeated pattern of a keystream; for example, if a keystream of $n$ bytes has no repeated pattern, then its period is equal to $\mathrm{n}$.

In the following simulations, we consider four different types of initial keystreams (Ms). Type-1 $\mathrm{M}$ is purely random, Type-2 M is of all 0's, Type-3 M is of all I's, and Typc-4 M consists of altemate 8-bit 0's and 8-bit I's. Four different types of input messages (nis) are also considered. Type- $1 \mathrm{~m}$ is purely random, Type- $2 \mathrm{~m}$ is of all 0 's, Typc-3 $\mathrm{m}$ is of all l's, and Type-4 mi consists of alternate 8-bit 0's and 8-bit l's. There are total $16(4 * 4)$ combinations (cases) with respect to Ms and ins.

\section{Randomness}

Two different sizes of Ms, 20480 bytes (Ml) and 20479 bytes (M2), are assumed. Note that 20480 is multiples of $256\left(2^{8}\right)$ where 20479 is not. The size of the input message is $2000 \mathrm{KBytcs}$. Each test case is run 100 times, and then the average is computed.

Tables 2(a) and 2(b) show the simulation results of Ml and $\mathrm{M} 2$, respectively.

Table 2(a). Simulation results of $\mathrm{Ml}$

\begin{tabular}{|c|c|c|}
\hline $\mathrm{Ml}>\mathrm{m}$ & Type-1 & Турс-2 \\
\hline Type-1 & $0.500033 / 0.499967$ & $0.500003 / 0.499997$ \\
\hline Typc-2 & $0.497006 / 0.502994$ & $0.503482 / 0.496518$ \\
\hline Туре-3 & $0.498209 / 0.501791$ & $0.485105 / 0.514895$ \\
\hline Type-4 & $0.497070 / 0.502930$ & $0.509102 / 0.490898$ \\
\hline & Гуре-3 & Type-4 \\
\hline Type-1 & $0.500003 / 0.499997$ & $0.500003 / 0.499997$ \\
\hline Typc-2 & $0.503482 / 0.496518$ & $0.503482 / 0.496518$ \\
\hline Туре-3 & $0.485105 / 0.514895$ & $0.485105 / 0.514895$ \\
\hline Type-4 & $0.509102 / 0.490898$ & $0.509102 / 0.490898$ \\
\hline
\end{tabular}

Lcgend: $a / b$, where a is the probability of being 0 , and $b$ is the probability of being 1

Table 2(b). Simulation results of $\mathrm{M} 2$

\begin{tabular}{|c|c|c|}
\hline $\mathrm{M} 2{ }^{\mathrm{m}}$ & Туре-1 & Турс-2 \\
\hline Type-1 & $0.500010 / 0.499990$ & $0.499985 / 0.500015$ \\
\hline Type-2 & $0.499929 / 0.500071$ & $0.508430 / 0.491571$ \\
\hline Type-3 & $0.500348 / 0.499652$ & $0.490608 / 0.509392$ \\
\hline Type-4 & $0.500144 / 0.499856$ & $0.497414 / 0.502586$ \\
\hline M2 & Тype-3 & T'ype-4 \\
\hline Type-1 & $0.499984 / 0.500016$ & $0.409987 / 0.500013$ \\
\hline Type-2 & $0.496909 / 0.503091$ & $0.500527 / 0.499473$ \\
\hline Type-3 & $0.487560 / 0.512440$ & $0.487418 / 0.512582$ \\
\hline Type-4 & $0.511040 / 0.488960$ & $0.497268 / 0.502732$ \\
\hline
\end{tabular}

Legend: $a / b$, where $a$ is the probability of being 0 , and $b$ is the probability of being 1

By cxamining Tablc 2(a), we notice that the probability of being 0 and that of being 1 are the same, for Typc-2 m, Type-3 $\mathrm{m}$, and Type-4 $\mathrm{m}$. This phenomenon indicates that the length of the initial keystream (M) should not be multiples of 256 , since the $\mathrm{C} 8$ algorithm processes data on a byte-by-byte basis. By observing Table 2(b), we find that the probability of being 0 and that of being 1 are close to one half, for ali test cascs. This phenomenon indicates that the $\mathrm{C} 8$ algorithm docs produce key strings of evenly distributed 0 's and 1 's, regardless of input patterns.

\section{Periol}

We consider four different pairs of $\left(l_{M}, l_{1: 1}\right)$, where $l_{M}$ and $l_{m}$ represent the length (the number of bytes) of the initial kcystream $\mathrm{M}$ and the input message $\mathrm{m}$, respectively. Those four pairs are (1200, 20480), (2400, 20480), (1200, 102400), and (2400, 102400). For Type-1 M and m, their period is equal to $I_{M}$ and $I_{m}$, respectivcly. For Type-2 $M$ and 
IIn, their period is equal to 1 . For Type-3 $M$ and $\mathrm{m}$, their period is equal to $\mathbf{l}$. For Type-4 $\mathrm{M}$ and $\mathrm{m}$, their period is equal to 2 .

Tables $3($ a) to $3(d)$ present the simulation results of pairs 1 to 4 , respectively.

Table 3(a). Keystream periods with respect to pair 1 (120., 20480)

\begin{tabular}{|c|c|c|c|c|}
\hline $\mathrm{M}$ & Type-1 & Type-2 & Type-3 & Type-4 \\
\hline Type-1 & 20480 & 20480 & 20480 & 20480 \\
\hline Type-2 & 20480 & 20480 & $\frac{20480}{20480}$ & $\frac{20480}{20480}$ \\
\hline Type-3 & 20480 & 20480 & 20480 & 2040480 \\
\hline Type-4 & 20480 & 20480 & 20480 & 20480 \\
\hline
\end{tabular}

Table 3(b). Keystreim periods with respect to pair 2 $(2400,20480)$

\begin{tabular}{|c|c|c|c|c|}
\hline $\mathrm{M}$ & Type-1 $^{\mathrm{m}}$ & Type-2 & Type-3 & Type-4 \\
\hline Type-1 & 20480 & 20480 & -20480 & 20480 \\
\hline Type-2 & 20480 & 20480 & 20480 & 20480 \\
\hline Type-3 & 20480 & 20480 & 20480 & 20480 \\
\hline Type-4 & 20480 & 20480 & 20480 & 20480 \\
\hline
\end{tabular}

Table 3(c). Keystream periods with respect to pair ? $(1200,102400)$

$\left[\begin{array}{c|c|c|c|c|}\hline \mathrm{m} & \text { Type-1 } & \text { Type-2 } & \text { Type-3 } & \text { Typc-4 } \\ \hline \text { Type-1 } & 102400 & 102400 & 102400 & 102400 \\ \hline \text { Type-2 } & 102400 & 102400 & 102400 & 102400 \\ \hline \text { Type-3 } & 102400 & 102400 & 102400 & 102400 \\ \hline \text { Typc-4 } & 102400 & 102400 & 102400 & 102400 \\ \hline\end{array}\right.$

Table 3(d). Keystrcam periods with respect to pair 4 $(2400,102400)$

\begin{tabular}{|c|c|c|c|c|}
\hline M & Type-1 & Type-2 & Type-3 & Type-4 \\
\hline Type-1 & 102400 & 102400 & 102400 & 102400 \\
\hline Type-2 & 102400 & 102400 & 102400 & 102400 \\
\hline Type-3 & 102400 & 102400 & 102400 & 102400 \\
\hline Type-4 & 102400 & 102400 & 102400 & 102400 \\
\hline
\end{tabular}

By examining Tables $3(\mathrm{a})$ to $3(\mathrm{~d})$, we notice that keystrcams generated by the $\mathrm{C} 8$ algorithm always maintain the maximal period, regardless of input patterus. This phenomenon indicates that the $\mathrm{C} 8$ algorithm is able to produce key strings with infinite period.

\subsubsection{Efficiency Analysis}

The C8 algorithm using only the XOR, INCREASE: and ADDITION operations is very efficient.

\subsection{Analysis of C.5}

The C5 aigorithm is based on strcam cipher. In our design, the simplest stream cipher using only the XOR operation [20] is recommended; thercfore, the CS algorithm is very efficient. As to the security of $\mathrm{C5}$, it is very sccure since the keystream $(\mathrm{K})$ generated by $\mathrm{C} \&$ has infinite period.

\subsection{Comparison}

Table 4 compares the architccture of today's GSM network with that of the proposed GSM network.

The current architecture uses the challenge-response mechanism (A3) for user authentication and key cxchange. In GSM standards, symmetric cryplography is recommended for supporting this mechanisn. Symmetric cryptography is faster than, but less secure than, public-key cryptography. If the A3 algorithm is revealed, the known-plaintext attack could be altempted during, user authentication. The publickey-based C3 protocol is more secure, but slower than the A3 algorithm

The period of the keystreate of the $\mathrm{CS}$ algorithm can be very long, which is ill sharp contrast to the fixed-period keystream of the As algorithm; the longer the period of the keystream, the securer the stream cipher. Although it takes more time to cxchange a longer keystream (M) during connection establishuent; however, stream cipher using only the $\mathrm{XOR}$ operation reduces the overheads during messages transfer. The CS algorithm can use any publicly available stream cipher whercas the A5 algorithun is preprictary.

Table 4. Comparison between the current architecture and the proposed architecture

\begin{tabular}{|c|c|c|}
\hline & $\begin{array}{l}\text { The Current } \\
\text { Architecturt: }\end{array}$ & $\begin{array}{l}\text { The froposed } \\
\text { Architcelure }\end{array}$ \\
\hline Complexily & $\begin{array}{l}\text { Authentication is fast } \\
\text { Key exchange is fast } \\
\text { Message cncryption is } \\
\text { fast }\end{array}$ & $\begin{array}{l}\text { Authentication is slow } \\
\text { Kcy exchange depends } \\
\text { on the key length } \\
\text { Messige encryption is } \\
\text { fast }\end{array}$ \\
\hline Siccurity & $\begin{array}{l}\text { Authentication is not } \\
\text { secure enough } \\
\text { Only the mobile user } \\
\text { is authenticated } \\
\text { Period is fixed and is } \\
\text { not very long }\end{array}$ & $\begin{array}{l}\text { Authentication is very } \\
\text { sccure } \\
\text { Both the mobile user } \\
\text { and the base station } \\
\text { are authenticated } \\
\text { (mutual } \\
\text { authentication) } \\
\text { Period can be infinite }\end{array}$ \\
\hline Flcxibility & $\begin{array}{l}\text { A3, A8 and AS are } \\
\text { proprictary } \\
\text { The SIM stores user's } \\
\text { personal } \\
\text { information and the } \\
\text { A3 algorithm }\end{array}$ & $\begin{array}{l}\text { CI, C8 and C5 are } \\
\text { publicly available } \\
\text { The SIM only stores } \\
\text { user's personal } \\
\text { information }\end{array}$ \\
\hline
\end{tabular}

\section{CONCLUSIONS}

\subsection{Summary}

In this paper, we focus on the sccurity of the Global 
System for Mobile communication (GSM) networks. Secure communication mechanisms for the GSM network are proposed. In the proposed architecture, we use public-kcy cryptography for uscr authentication, and stram cipher for message encryption and decryption. Cryptanalysis and operational analysis show that the $\mathrm{C} 3$ protocol is sccurc and efficient. Simulation results indicate that the key gencration method can always produce key strings of evenly distributed 0 's and l's and with infinite period. Those security mechanisms significantly improve the security of today's GSM nctwork.

\subsection{Future Works}

A very important part of the proposed architecture is the C5 algorithm. Although any publicly available stream cipher can be used; lowever, the effectiveness and efficiency of the selected stream cipher significantly affect the performance and the security of GSM networks. In the future research, we will survey existing stream ciphers; for example, the pscudorandom bit generator with bilateral step control [20], and design a more effective and efficiont stream ciphor for GSM networks.

\section{REFERENCE}

(1) Bruce Sohneier, "Applied cryptography: Protocois, algorithms, and sourec code in C", Wiley

12) R. Anderson and R. Needhan, "Rohushess Principles for Public Kisy Protocols". Research Notes

[3] David G.W. Birch and lan J. Shaw, "Mobile Conmuncications Security Private or Public", IlEE, June 1994

|4 R. Bird et al., "Systematic Design of a Jamily if Altack-Resistant Authentication Protocols", IF.EE Journal on selected Areas in Communications. Vol. 11, No.5, Jume 1993

[5] R. Lircl ct al., "Systematic Desien of Truo-l'anty Authentication Protocols", Advatiess in Cryptology - CRYP'TO '91, $1991, p$ p.44-61

(6) Charles Brookson, "GSN Security: A deseription of the reasons for security and the techniques", IE: 1994

[7] J.C. Cooke and R.L. Brewster, "Cryplograplic security technicpues for digital mobile telephones", Wireless Commtnications, 1992

[81 D.E. Denning and G.M. Sacco, "Timestamps in Key Distribution Protocols", Communications of the ACM, Vol.24, No.8, pp.533-536, Dec. 1981

19] L. Gong. "Filicient Network Authentication Protocols: lower bounds and optimal implementations", Distributed Computing, July 1995, pp.131145

[10 Clarle Kaufman, Rada Perlamn and Wike Speciner, "Network Security: PRIVATE Communication in a I'UHI,IC World", Pentice Hall, 1995

[11] Yi-Bing Lin, "No wires attached", IEEE Potentials, Vol. 14, Oct. 1995

[12] Jianwei Liu and $Y$ umin Wang. "A user authentication protocol for digital mobile communication network", PIMRC'95, Vol. 2. 1995

[13] Jianwei Liu and Yumin Wang, "Authentication of mobile users in personal comnunication system", PIMRC'96, Vol. 3, 1996

[14] Chi-Chun L,o and Yi-Chun Yel, "Authentication Protocols for the Broadband ISDN Billing Systen", subnitted to Computer Standards \& Interfaces

[15] A Mehrotra and L.S. Goidting, "Mobility and security management in the GSM system and sonse proposed future jmprovements", Proceedings of the IEEE, Vol. 86, Issue 7, July 1998

[16] M. Meuly and M.-E3. Pautet, "GSM Protocol Architecture: Radio Sub. system Signalling", IFEF, 4 l st Vehicular Technoløgy Conference, 1991

[17] M. Mouly and M.-E3. Pautet, "The GSM System for Mobile Commonications", 1992

[18] John Scourias, "A Brief Overview of GSM", http://kbs.cs.tuberlin.de/ jutta/gsm/js-intro.htmul, 1994

[19] S.J. Stueptuerd, "Public key stream ciphets", IF.E. 1994

[20 Kencheng Zeng et al, "Pseudorandom Bit Cienerators in Stream-Cipher Cryptography", IFEF; Computer, 1991

[21] ITU Recommendation X.509(1993E)|ISO/LEC 9594-8 Intornation
Technology-Open Systenss [ntercontlution + The Directory: Aulhentication Framework. ITU-T SG7|[SO/ILC JTCI/SC21/WG4. 1993 